\title{
Estrategias para la internacionalización de la educación superior
}

\author{
Strategies for internationalization of higher education
}

Ana Esperanza Burgos Castellanos ${ }^{1}$ y Rosabel Torrellas Hidalgo ${ }^{2}$

${ }^{1}$ Universidad Nacional de Colombia. ${ }^{2}$ Universidad Distrital Francisco José de Caldas aeburgosc@unal.edu.co y rosabel.torrellas@gmail.com

\section{Resumen}

La educación superior en el contexto mundial es un elemento estratégico que propicia la integración y el intercambio del conocimiento dentro de los acelerados cambios económicos, políticos, culturales y sociales de las sociedades modemas. Las instituciones de educación superior deben estar convencidas de su responsabilidad social frente al desarrollo humano, la construcción de la paz, la justicia y el progreso. La educación modema debe orientarse hacia la profundización del conocimiento científico y humanístico para alcanzar la comprensión compleja de las problemáticas mundiales frente a las locales y regionales y poder dar así respuesta a los agobiantes problemas que afectan a la humanidad de hoy.

\section{Palabras clave}

Internacionalización de la educación superior, tecnologías de la información y la comunicación, educación a distancia.

\section{Abstract}

Higher education in the global context is a strategic element that facilitates the integration and interchange of knowledge within the accelerated economic, political, cultural and social aspects of modem societies. Higher education institutions must be convinced of its social responsibility to human development, the establishment of peace, justice and progress. Modem education should be oriented towards the deepening of scientific and humanistic knowledge to achieve understanding of complex global problems compared to local and regional and be able to respond to the pressing problems affecting humanity today.

\section{Keywords}

Internationalization of higher education, information technology and communication, distance learning.

\section{Introducción}

El presente trabajo constituye una reflexión acerca de la importancia de la internacionalización de la educación superior, la visión en el contexto internacional, la descripción de diversos enfoques sobre la intemacionalización y un análisis del por qué es importante intemacionalizar la educación superior. 


\section{Desarrollo}

La internacionalización de la educación tiene diferentes significados que a menudo se confunden con el de globalización. La visión de la educación superior en el contexto mundial establece la integración de una dimensión internacional e intercultural en las funciones de docencia, investigación y extensión que contribuyan a mejorar la calidad de vida de la humanidad (UNESCO, 1998).

De otra parte, la globalización es un concepto complejo ampliamente debatido por una diversidad de autores y susceptible de ser abordado desde muy diversas perspectivas. En términos generales se refiere a un proceso histórico, básicamente económico que tiende a la integración de las economías nacionales en una única economía de mercado mundial, mediante el flujo de tecnología, conocimientos, personas, valores e ideas a través de las fronteras y afecta a cada país de manera diferente en virtud de sus caractenísticas culturales e histónicas.

En este contexto, la intemacionalización de la educación superior es la respuesta de un país para enfrentar las repercusiones de la globalización mediante la integración internacional e intercultural de la enseñanza, la investigación y la extensión. Así, la globalización y la internacionalización de la educación son dos conceptos, aunque distintos, vinculados activamente (García, 2005).

La dimensión intemacional de los programas se apoya en el sistema de créditos académicos, para facilitar la convalidación de los títulos y proporcionar la comparación y homologación de los estudios realizados en diversas instituciones. Considerando los aspectos anteriores, la primera etapa necesaria para iniciar el proceso de internacionalización de la educación corresponde a la revisión profunda de los perfiles profesionales y laborales de las carreras que se dictan en una institución y su comparación con los estándares internacionales y las tendencias mundiales.

Luego de realizado este análisis se debe proceder a la evaluación exhaustiva de los programas, los contenidos de sus materias, el número de créditos y las competencias desarrolladas, entre otros aspectos para adecuarlos a los estándares internacionales que permitirán la factibilidad de su convalidación internacional sin difiaultad. La coherencia y planificación de este proceso, junto con las estrategias y la dotación de los recursos económicos necesarios, hará posible consolidar la internacionalización de los programas académicos de una institución.

Otro aspecto importante a considerar en esta etapa, es la instauración de plataformas virtuales en las instituciones que permitan ofrecer programas de pregrado y postgrado mediante educación a distancia. Una vez estructurados los criterios anteriores para internacionalizar los programas y contando con una robusta plataforma virtual puede pasarse a la segunda etapa que permitirá racionalmente ofrecer programas académicos que sean competitivos en el ámbito intemacional y desarrollar la proyección de la institución. Demás está decir, que la calificación y experiencia de los docentes es un aspecto crítico a la hora de internacionalizar los programas académicos.

La mayoría de las universidades del mundo requieren que sus profesores tengan estudios de doctorado y amplia experiencia docente universitaria. Por ello, ante la posible ausencia de docentes calificados, las universidades actualmente contratan profesores extranjeros con las credenciales necesarias para el dictado de los cursos a distancias desde sus países de origen, utilizando los beneficios que ofrecen las Tecnologías de la Información y la Comunicación (TICs) (Enníquez, 2006). Otras estrategias necesarias para intemacionalizar los programas académicos de una institución de educación superior deben contemplar una serie de actividades que faciliten realizar convenios de cooperación internacional entre la institución y organizaciones públicas y privadas, establecer planes de intercambio estudiantil, induir el estudio de un idioma extranjero, fomentar estudios o trabajos en el extranjero y propiciar la visita de estudiantes de otros países. 
De otra parte, es necesario que se desarrollen programas conjuntos de maestría y doctorado, se incentive la movilidad del personal académico y administrativo, así como de profesores visitantes y conferencistas y se vinculen los programas académicos con la investigación y la capacitación. Además, la internacionalización de la investigación requiere organizar proyectos de investigación con universidades extranjeras y desarrollar investigaciones conjuntas, propiciar conferencias y seminarios internacionales, impulsar la publicación de artículos y ensayos y buscar el apoyo financiero y académico internacional. Asimismo, es necesario crear programas de intercambio de estudiantes de postgrado, docentes e investigadores que apoyen los procesos de desarrollo cultural y fomenten valores universales vinculados al respeto por la dignidad humana, la equidad, la tolerancia y la búsqueda del desamollo humano y social, entre otros.

Igualmente, es importante fortalecer la articulación de la investigación y los contenidos de los programas académicos, como también las relaciones y los servicios extemos establecidos entre el país y el extranjero. En consecuencia, se requiere la organización de proyectos internacionales para el fomento del desarrollo, definir localidades de enseñanza fuera de las fronteras mediante la educación a distancia, crear redes internacionales de participación y programas para alumnos en el extranjero mediante la plataforma virtual de la institución. De igual forma, las actividades extracurriculares deben considerar la creación de asociaciones de estudiantes, intercambios internacionales y la organización de grupos de estudio para el conocimiento de otras culturas y lenguas.

Además, debe contemplarse la participación activa del personal académico y administrativo de la institución para facilitar la planificación, la estimación del presupuesto y la revisión de los sistemas de calidad en la institución por facultades y programas académicos. Por tanto, se requieren estucturas organizacionales modernas, sistemas de comunicaciones efectivas y los recursos financieros necesarios. Los servicios de apoyo deben facilitar el alojamiento de estudiantes, organizar cursos de idiomas para fomentar el intercambio cultural, brindar ayuda a los estudiantes extranjeros que asisten a la universidad y a los nacionales que van al extranjero.

Finalmente, los procesos que contribuyen a posicionar una institución en el ámbito académico internacional requieren principalmente el desarrollo del talento humano, la consolidación de políticas de compensación y promoción para estimular la participación del personal académico y administrativo y el apoyo de intercambios con el extranjero, ya sea de años sabáticos, pasantías, entre otros.

\section{Conclusiones}

La educación superior en el contexto mundial es la fuente principal de integración internacional e intercultural que contribuye al progreso de la humanidad y promueve la interacción entre los países dentro de los cambios que caracterizan las sociedades modemas.

La internacionalización de la educación superior es un proceso que reconoce a la ciencia, la tecnología y la innovación como instrumentos fundamentales para el desarrollo económico, social y político de los países.

Los programas académicos de la educación intemacional deben incluir el dominio de otras lenguas, profundizar los contenidos de las áreas de las ciencias básicas y de la tecnologías de la información y la comunicación, la comprensión compleja de las problemáticas mundiales frente a las locales y regionales, los temas de paz, justicia, dignidad y equidad, resolución de conflictos y desarrollo sostenible. 
Las directivas de las instituciones de educación superior deben tener una actitud proactiva para liderar el proceso de internacionalización a través de políticas y programas integrados a la vida de la institución.

Los procesos de intemacionalización de la educación superior requieren un conjunto de estrategias para generar programas educativos competitivos acordes con los estándares internacionales.

Las Tecnologías de la Información y Comunicación (TICs) facilitan los procesos de internacionalización de la educación.

\section{Bibliografía}

Enríquez C. J. (2006). Educación superior: Tendencias y Desafíos. Educ. méd. 9 (1), 6-10.

García, G. C. (2005). Complejidades de la globalización e intemacionalización de la educación superior: Interrogantes para América Latina. CDC. 22 (58), 1-22.

UNESCO. (1998). La educación superior en el siglo XXI: visión y acción. Conferencia Mundial sobre la Educación Superior. Documento de trabajo. Pańs. [En línea] uww. unesco.org/education/educprog/wche/dedaration_spa.htm - 78k 